Check for updates

Cite this: RSC Adv., 2018, 8, 36286

\title{
Preparation of novel phosphorus-nitrogen-silicone grafted graphene oxide and its synergistic effect on intumescent flame-retardant polypropylene composites
}

\begin{abstract}
Gaowei Yuan, ${ }^{\text {ab }}$ Bing Yang, ${ }^{a}$ Yinghong Chen (D)*a and Yinggang Jia (D) ${ }^{b}$
Due to the poor dispersion in polymer matrix, graphene can hardly be used alone as a flame-retardant additive for polymers. In this paper, a novel halogen-free flame retardant - the ternary graft product of silsesquioxane, graphene oxide and 9,10-dihydro-9-oxa-10-phosphaphenanthrene-10-oxide (PMGO) with a structure of graphene oxide layers modified with the synergistic flame retardant multiple elements of phosphorous, nitrogen and silicon was synthesized and then used in combination with traditional intumescent flame retardant (IFR) to enhance the flame retardancy of polypropylene (PP). The experimental results show that the thermal and flame retardant properties of flame-retardant (FR) PP composites are significantly improved by introducing $5 \mathrm{wt} \%$ PMGO as well as $20 \mathrm{wt} \%$ IFR. The peak heat release rate and total heat release of the FR PP composite are reduced $61.5 \%$ and $40.2 \%$, respectively, compared to neat PP. Based on the char layer observation and thermal analysis, the enhancement in flame retardancy is mainly attributed to the outstanding intumescent char layers with high strength and thermal stability formed under the synergistic effect of PMGO and IFR. Besides, the introduced phosphorous, nitrogen and silicon hydrophilic groups do not show the negative effects on the surface hydrophobicity of flame retardant PP materials, which could broaden its scope of application.
\end{abstract}

Received 5th September 2018
Accepted 20th October 2018

DOI: $10.1039 / c 8 r a 07418 f$

rsc.li/rsc-advances
In the past few decades, due to the low smoke release, absence of halogens and no melt dropping properties, halogen-free intumescent flame retardants (IFRs) have become the primary strategy in flame-retarding of polymers especially polyolefins. ${ }^{13-15}$ Generally, the conventional IFRs system includes three elementary ingredients: the acid source, the carbonization source and the blowing agent. ${ }^{16} \mathrm{~A}$ mixture of ammonium polyphosphate (APP), pentaerythritol (PER) and melamine (MEL) is undoubtedly by far the most representative halogen-free IFR system, which could produce a swollen and compact char layer functioning like a barrier against heat and mass transfer between the flame zone and the pyrolysis front within the polymeric materials. ${ }^{17}$ Herein, the compact char layer is formed through the esterification and cross-linking interaction between acid source and charring agent, which can swell and expand under the inert gases release from the thermal decomposition of blowing agent. ${ }^{14,18}$ Although a higher-level fire resistance has been achieved, the major problems encountered with the above system are the high loading required and the poor water resistance.

Due to the extremely high surface area as well as fantastic electrical, thermal, and mechanical properties, graphene has attracted many interests for a large variety of potential applications. ${ }^{19-24}$ In recent years, a lot of literatures have reported the use of graphene as synergistic flame retardant nanofiller for polymer flame retardancy. This is mainly based on its intrinsic 
fire resistance and unique layered structure. ${ }^{\mathbf{1 2 , 2 5 , 2 6}}$ However, the big challenge of graphene application could be in the strong van der Waals interactions between graphene layers making them tend to be easily agglomerated and this surely limits their wider applications..$^{27-29}$ On the other hand, the related research shows that the pure bare graphene is easily burnt out under air atmosphere. As a result, the only graphene incorporated polymer system could not form the good charred layers as an effective barrier to inhibit the diffusion of flammable lowmolecular-weight decomposition products. ${ }^{26}$ In order to tackle this problem, some chemical modifications can be considered to be carried out on graphene nanosheets. Through this way, some flame retardant elements can be purposefully introduced into the graphene skeleton because on the surface and edge of the precursor of graphene-graphene oxide (GO), there are large amount of oxygen functional groups (such as epoxy, hydroxyl and carboxyl groups), which can provide the reactive sites. ${ }^{30-32}$ Phosphorus, nitrogen and silicon compounds are efficient flame quenching materials and radical scavengers. In addition, the nitrogen is also the environmental friendly element. It can be predicted that the flame retardant structures and elements such as silsesquioxane (POSS) and organic phosphorus to be anchored on the surface of graphene (phosphorus/nitrogen/ silicon functionalized GO) can not only facilitate the dispersion of graphene but also help to form some solid and tough char layers during combustion. This helpfully broadens the applications of polymer/graphene nanocomposites ${ }^{33-37}$ for fire retardancy. On the other side, it is also very interesting to investigate the synergistic flame retardancy effect between IFR and phosphorus/nitrogen/silicon functionalized GO in polymer materials.

Taking into account above problems, we have successfully synthesized a novel multi-element synergistic flame retardant (PMGO) by grafting silicone-nitrogen elements based cage octaminopropyl silsesquioxane (oapPOSS) and phosphorus element based 9,10-dihydro-9-oxa-10-phosphaphenanthrene10-oxide (DOPO) on the nanolayers of graphene oxide. In this paper, we focused on the synergism between the prepared PMGO as a synergist and the intumescent flame retardant (IFR) we prepared priorly in flame retarding of PP. The synergistic effects of PMGO on IFR incorporated PP composite in improving the quality of the char layers formed during combustion and hence the flame retardancy were deeply investigated based on the thermogravimetric analysis (TGA), Xray photoelectron spectroscopy (XPS), scanning electronic microscope (SEM) observation and flammability tests. Besides, the possible thermal degradation and FR mechanism and wettability of this novel FR system were also investigated. It is expected that the combination of PMGO and IFR could improve the thermal stability, flame retardancy and hydrophobicity properties of FR PP materials.

\section{Experiments}

\subsection{Materials}

Polypropylene (PP), a commercial T30 s polymer, used in this work was purchased from China Petroleum \& Chemical
Corporation Lanzhou Petrochemical Company. The modified IFR (APP-PEL-PER) from the thermal reaction product of APP, PER and MEL, octaminopropyl silsesquioxane and graphene oxide were prepared priorly in our laboratory. DOPO with $98 \%$ purity was obtained from Aladdin Biochemical Technology Co., Ltd (Shanghai, China). All solvents including $N, N$-dicyclohexylcarbodiimide (DCC), methanol, acetone and tetrahydrofuran (THF) were analytical grade and provided by Sinopharm Chemical Reagent Co., Ltd (Shanghai, China).

\subsection{Preparation of PMGO FR synergist}

2.2.1 Synthesis of phosphorus contained graphene oxide. Phosphorus contained graphene oxide was prepared by reaction of $\mathrm{P}-\mathrm{H}$ group in DOPO with epoxy functional groups in GO. A certain amount of the as-prepared graphene oxide (about 600 $\mathrm{mg}$ ) was first suspended in THF and then treated for $30 \mathrm{~min}$ in an ultrasonic bath. The obtained suspension was transferred into a three-neck flask. Then, another THF solution containing DOPO ( $5 \mathrm{~g})$ was added under stirring. The mixture was heated to $75{ }^{\circ} \mathrm{C}$ and refluxed for $12 \mathrm{~h}$ under $\mathrm{N}_{2}$. After the functionalization process, the mixture was separated by filtration and thoroughly washed with anhydrous THF and acetone to remove the residual DOPO. Finally, the reaction intermediate products were dried under vacuum at $80{ }^{\circ} \mathrm{C}$ overnight to remove the solvent and the corresponding yield is about $400 \mathrm{mg}$.

2.2.2 oapPOSS grafted phosphorus containing graphene oxide (PMGO). A certain amount of the modified graphene (about $200 \mathrm{mg}$ ) was initially dispersed in THF and sonicated for $2 \mathrm{~h}$. Then, oap-POSS ( $2 \mathrm{~g}$ ) and DCC (100 mg) were added to the resultant suspension, followed by sonication for another $30 \mathrm{~min}$. The mixture was then well mixed and refluxed for $48 \mathrm{~h}$ at $75{ }^{\circ} \mathrm{C}$ under nitrogen atmosphere. Then, the solution was vaccum-filtered through a PTFE micrometer membrane and washed three times with anhydrous methanol and THF. Finally, the obtained solid was dried under vacuum at $80^{\circ} \mathrm{C}$ overnight to remove the solvent and its corresponding yield is about $300 \mathrm{mg}$. The synthesis route of PMGO is shown in Scheme 1.

\subsection{Preparation of FR PP composites}

$\mathrm{PP}$, IFR and PMGO were dried at $60{ }^{\circ} \mathrm{C}$ under vacuum for $8 \mathrm{~h}$ before used. Then, the mixture of above components with different ratio was mixed using an internal mixer (SU 70-1, Suyan Technological Company, China) at $60 \mathrm{rpm}$ and $190{ }^{\circ} \mathrm{C}$. The well compounded composites were hot-pressed at $190{ }^{\circ} \mathrm{C}$ into sheets with suitable size in a press vulcanizer for various tests including thermal behavior and flame retardant property. The composition of various FR PP sample is shown in Table 1.

\subsection{Measurements and characterizations}

Fourier-transform infrared spectra (FT-IR) characterization was performed on a Perkin Elmer-283B FT-IR Spectrometer and recorded between 400 and $4000 \mathrm{~cm}^{-1}$. The samples were grinded with $\mathrm{KBr}$ and pressed into pellets. Thermogravimetric analysis (TGA) was performed on a TGA Q5000IR thermoanalyzer (TA Instruments Inc., USA) from room temperature to $700{ }^{\circ} \mathrm{C}$ at a heating rate of $10{ }^{\circ} \mathrm{C} \mathrm{min}{ }^{-1}$ under nitrogen and air 


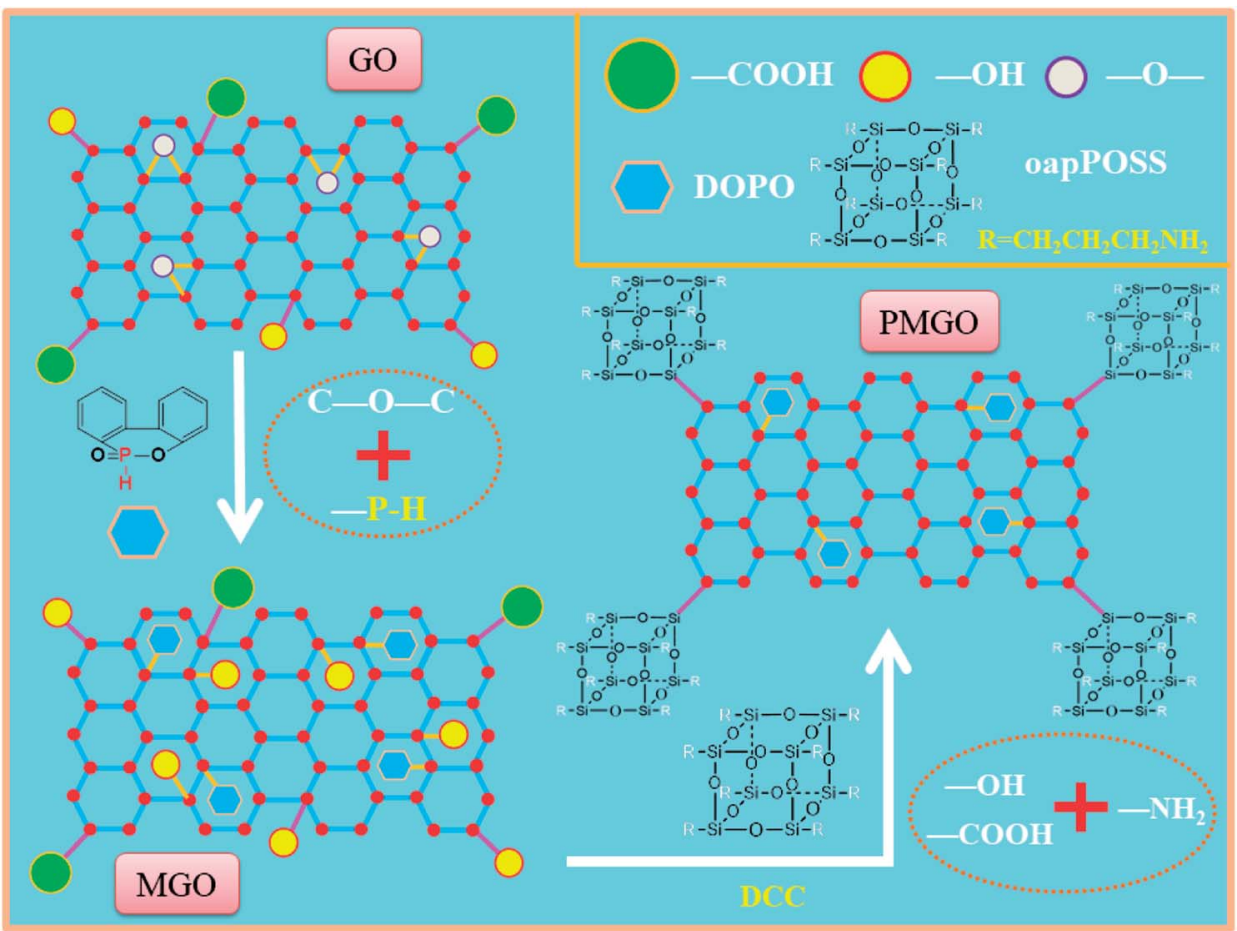

Scheme 1 The synthetic route and structure of PMGO.

Table 1 Formulations of FR PP composites

\begin{tabular}{lccl}
\hline Sample & PP $(w t \%)$ & IFR $(w t \%)$ & PMGO (wt $\%)$ \\
\hline PP0 & 100 & 0 & 0 \\
PP1 & 80 & 20 & 0 \\
PP2 & 75 & 25 & 0 \\
PP3 & 75 & 24 & 1 \\
PP4 & 75 & 22 & 3 \\
PP5 & 75 & 20 & 5
\end{tabular}

atmosphere. X-ray photoelectron spectroscopy (XPS) was performed on a VG Escalab Mark II spectrometer (Thermo-VG Scientific Ltd., UK) using $\mathrm{Al} \mathrm{K} \alpha$ excitation radiation $(h \nu=$ $1486.6 \mathrm{eV})$. Sample morphologies were observed using a scanning electronic microscope (SEM, Hitachi S-570). All the samples were sputter-coated with a thin gold layer (about 10 $\mathrm{nm}$ ). Micro-scale combustion calorimetry (MCC) measurement was conducted on a micro-scale combustion calorimeter (FAAPCFC, Fire Testing Technology Ltd., UK). About $2 \mathrm{mg}$ powder scraped from FR PP sample was heated from the ambient temperature to $800{ }^{\circ} \mathrm{C}$ at a heating rate of $1{ }^{\circ} \mathrm{C} \mathrm{s}^{-1}$ under air atmosphere. Limiting oxygen index (LOI) was measured using a HC-2 oxygen index meter (Jiangning Analysis Instrument Company, China) according to the standard ASTM D2863-2010. The dimension of samples used was $100 \times 6.5 \times 3 \mathrm{~mm}$. UL94 vertical burning test was conducted on a CZF-II horizontal and vertical burning tester (Jiangning Analysis Instrument Company, China) according to the standard ASTM D3801-2010. The dimension of samples used was $125 \times 12.5 \times 1.6 \mathrm{~mm}$. Angle Goniometer Sindatek Model DSA25 (Germany Kluwer company) was employed to measure the water contact angle by the sessile drop method with a micro-syringe at $25{ }^{\circ} \mathrm{C}$. More than 10 contact angles were averaged to get a reliable value for each sample.

\section{Results and discussion}

\subsection{FT-IR and XPS characterizations of PMGO}

Fig. 1 shows the FT-IR spectrum of the synthesized PMGO. For purpose of comparison, the FT-IR spectra of the raw materials DOPO, GO and oapPOSS are also provided in the same figure. It can be seen that the disappearance of C-O-C band at $1226 \mathrm{~cm}^{-1}$ (attributed to $\mathrm{GO}$ ) and $\mathrm{P}-\mathrm{H}$ band with lower frequency at $2436 \mathrm{~cm}^{-1}$ (attributed to DOPO) is observed in the spectrum of

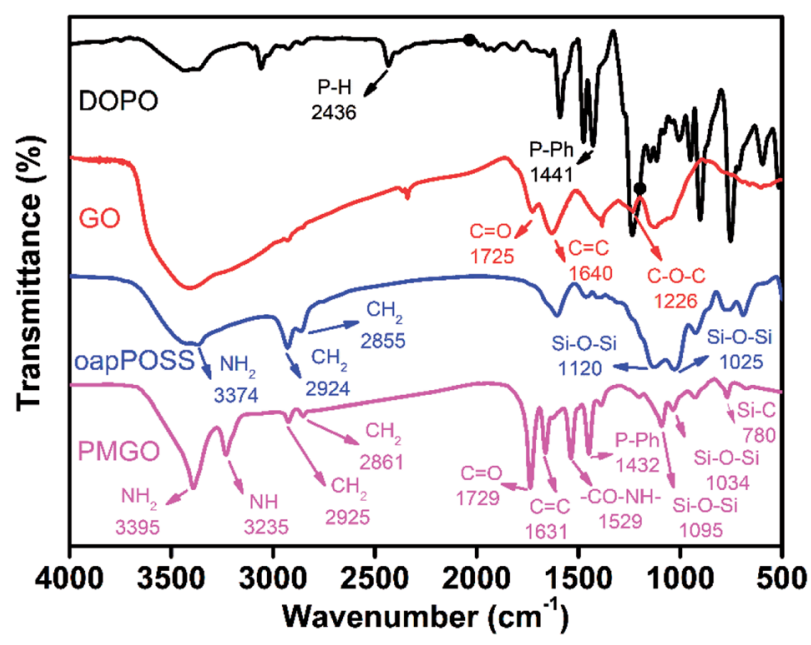

Fig. 1 FT-IR spectra of DOPO, GO, oapPOSS and PMGO. 

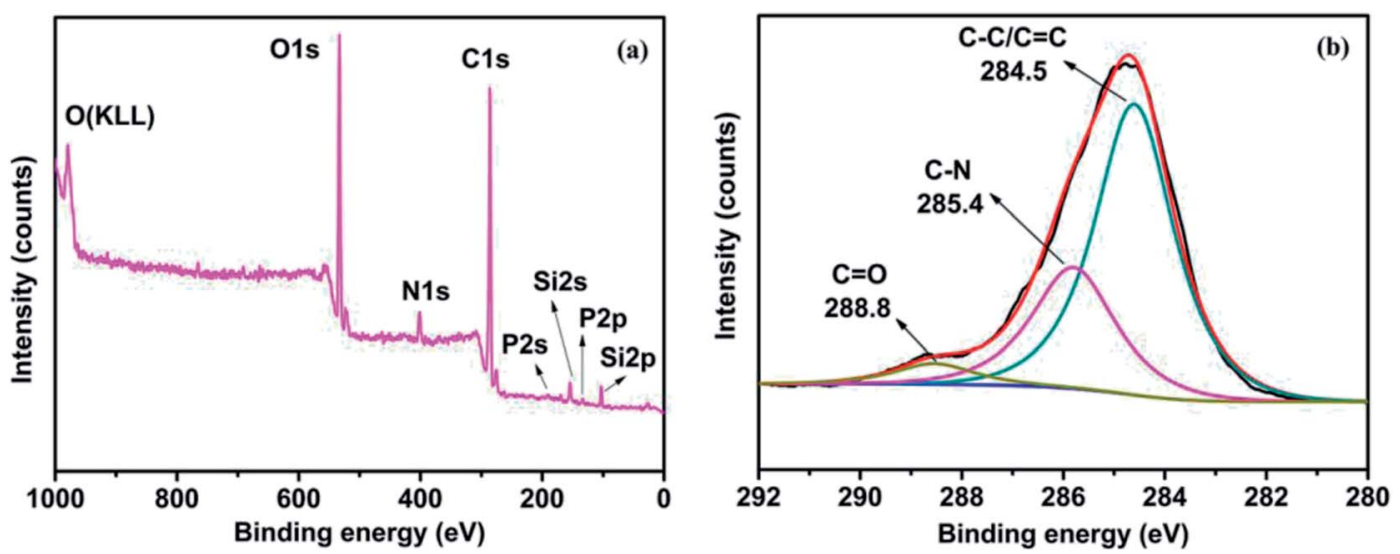

Fig. 2 The XPS survey spectrum of PMGO (a) and its deconvoluted high-resolution C1s spectra (b).

the final reaction product PMGO. In addition, the related peaks at $1432 \mathrm{~cm}^{-1}(\mathrm{P}-\mathrm{Ph})$ in DOPO, $1729 \mathrm{~cm}^{-1}(\mathrm{C}=\mathrm{O})$ and $1631 \mathrm{~cm}^{-1}$ $(\mathrm{C}=\mathrm{C})$ in $\mathrm{GO}$ still appear in the spectrum of PMGO. These results confirm the successful grafting of DOPO onto GO surface through the chemical reaction of the $\mathrm{P}-\mathrm{H}$ group with the epoxy ring:.$^{35}$ A sequence of characteristic absorption peaks of oapPOSS are observed at $3374 \mathrm{~cm}^{-1}$ (N-H stretching vibration) and $2924-2855 \mathrm{~cm}^{-1}$ (C-H stretching vibration). The intense absorption peak at $1120 \mathrm{~cm}^{-1}$ is the characteristic vibration of an $\mathrm{Si}-\mathrm{O}-\mathrm{Si}$ bond and the absorption peak at $1025 \mathrm{~cm}^{-1}$ is attributed to the special characteristic vibration of the silsesquioxane cage $\mathrm{Si}-\mathrm{O}-\mathrm{Si}$ framework. ${ }^{38}$ It is also noted that the spectrum of the resultant PMGO shows a strong absorption of $\mathrm{Si}-\mathrm{O}-\mathrm{Si}$ vibration at 1095 and $1034 \mathrm{~cm}^{-1}$ from oapPOSS structure, and the new peaks at $3235(\mathrm{NH})$ and $1529 \mathrm{~cm}^{-1}(\mathrm{CO}-\mathrm{NH})$ in PMGO, which is due to the dehydration reaction between the amino groups of oapPOSS and the carboxyl/hydroxyl group of DOPO modified GO. All these evidences demonstrate the oapPOSS has been grafted onto the surface of DOPO modified GO successfully and the PMGO has been obtained.

Fig. 2 shows the XPS survey spectrum of PMGO and the corresponding high-resolution C1s spectra. It can be seen that there are the extra peaks attributed to $\mathrm{P}, \mathrm{N}$ and $\mathrm{Si}$ observed in the spectrum of PMGO after grafting of DOPO and oapPOSS. In
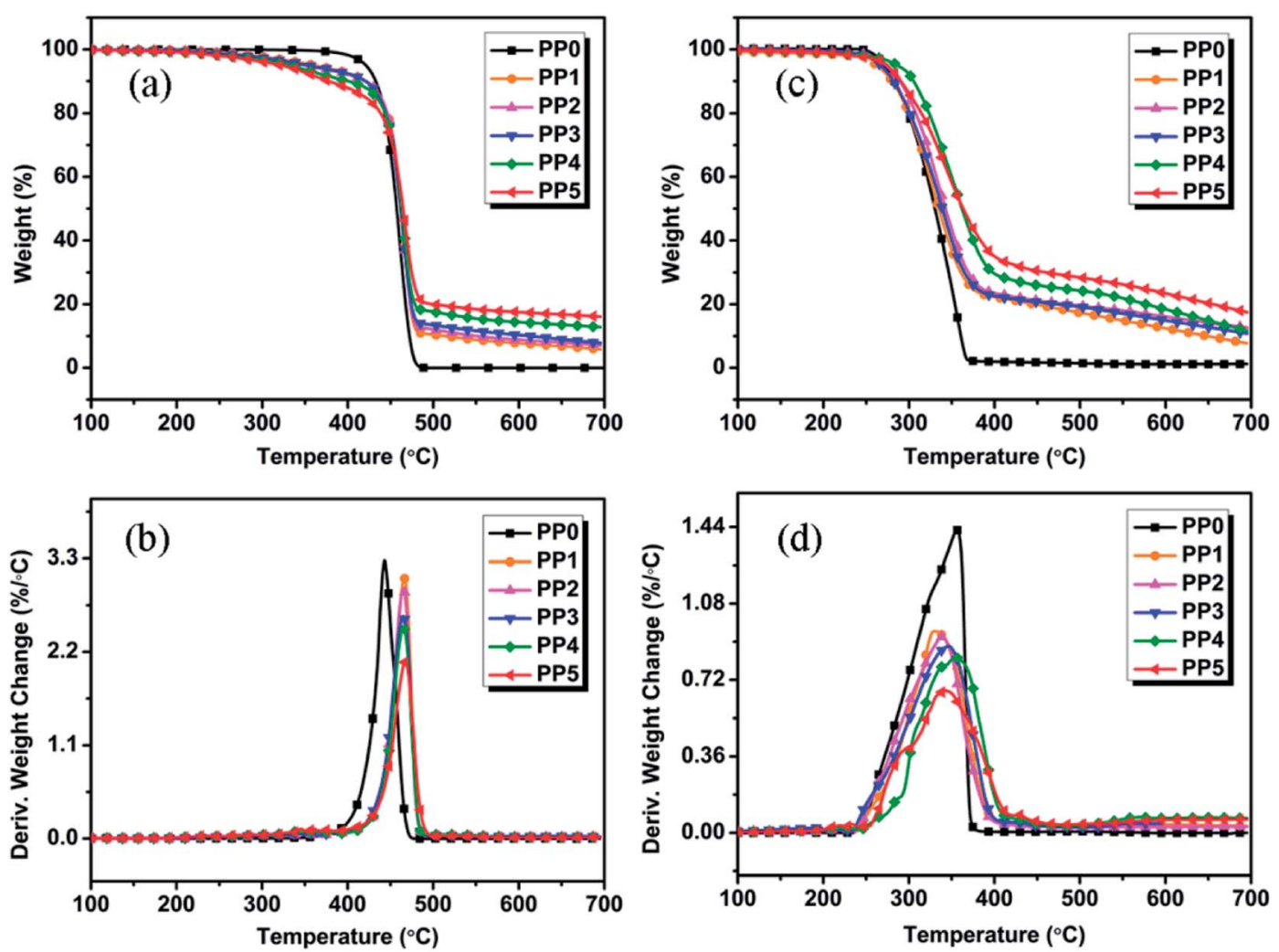

Fig. 3 TGA and DTG curves of FR PP composites under nitrogen atmosphere ( $a$ and $b$ ) and air atmosphere (c and d) at a heating rate of $10{ }^{\circ} \mathrm{C} \min ^{-1}$. 
addition, in the high-resolution C1s spectrum of PMGO, three peaks can be distinguished: $284.5 \mathrm{eV}$ is attributed to $\mathrm{C}-\mathrm{C}$ and $\mathrm{C}=\mathrm{C}$ in the $\mathrm{GO}$ skeleton, $285.4 \mathrm{eV}$ is attributed to $\mathrm{C}-\mathrm{N}$ (oapPOSS) and $288.8 \mathrm{eV}$ is attributed to $\mathrm{C}=\mathrm{O}$ (GO). ${ }^{33,34}$ The XPS analysis further confirms the chemical grafting of DOPO and oapPOSS onto GO surface.

\subsection{Thermogravimetry and morphology analysis of FR PP composites}

The weight and weight loss rate curves of all formulations obtained from TGA experiments conducted under nitrogen and air atmosphere are shown in Fig. 3. The corresponding thermal decomposition parameters of samples are included in Table 2 (nitrogen atmosphere) and Table 3 (air atmosphere). Unfortunately, no char residue is left for pure PP, illustrating the complete decomposition and inflammable property of PP when exposed to intense heat. The flame retarding mechanism of traditional IFR (PP2) tends to form the tender and flimsy expanded char layers in condensed phase, e.g. PP2 FR sample without synergist shows an enhanced yield of residual char (with $7.12 \mathrm{wt} \%$ ) relative to pure PP, and however, it still fails to achieve any flame retardancy level even at 25 wt $\%$ FR loading. These facts demonstrate that it cannot effectively improve the flame retardancy of PP composites by using IFR only alone. Compared with pure PP (PP0), the FR PP composites show the lower $T_{\text {onset }}$ values and the higher $T_{\max }$ values. Under nitrogen atmosphere, both FR PP composites and pure PP exhibit the

Table 2 The TGA parameters of FR PP composites under nitrogen atmosphere $^{a}$

\begin{tabular}{llllll}
\hline & & & \multicolumn{3}{c}{ Residual weight (\%) } \\
\cline { 5 - 6 } Sample & $T_{\text {onset }}\left({ }^{\circ} \mathrm{C}\right)$ & $T_{\max }\left({ }^{\circ} \mathrm{C}\right)$ & $500{ }^{\circ} \mathrm{C}$ & $600{ }^{\circ} \mathrm{C}$ & $700{ }^{\circ} \mathrm{C}$ \\
\hline PP0 & 406.6 & 443.4 & 1.68 & 0.85 & 0.28 \\
PP1 & 360.3 & 466.2 & 10.42 & 7.73 & 5.82 \\
PP2 & 350.4 & 464.6 & 12.06 & 8.83 & 7.12 \\
PP3 & 353.4 & 466.5 & 13.51 & 10.29 & 7.86 \\
PP4 & 332.9 & 466.9 & 17.68 & 14.40 & 12.83 \\
PP5 & 322.5 & 470.5 & 20.05 & 17.55 & 16.10
\end{tabular}

${ }^{a} T_{\text {onset }}$ is the temperature at $5 \%$ weight loss and $T_{\max }$ is the temperature at maximum rate of weight loss.

Table 3 The TGA parameters of FR PP composites under air atmosphere $^{a}$

\begin{tabular}{llllll}
\hline & & & \multicolumn{3}{c}{ Residual weight (\%) } \\
\cline { 5 - 6 } Sample & $T_{\text {onset }}\left({ }^{\circ} \mathrm{C}\right)$ & $T_{\max }\left({ }^{\circ} \mathrm{C}\right)$ & $500{ }^{\circ} \mathrm{C}$ & $600{ }^{\circ} \mathrm{C}$ & $700{ }^{\circ} \mathrm{C}$ \\
\hline PP0 & 271.4 & 357.4 & 0 & 0 & 0 \\
PP1 & 272.9 & 330.6 & 17.36 & 12.45 & 7.87 \\
PP2 & 266.8 & 339.7 & 19.47 & 16.07 & 12.65 \\
PP3 & 261.2 & 346.8 & 19.27 & 14.92 & 10.81 \\
PP4 & 286.3 & 354.5 & 24.39 & 18.40 & 11.66 \\
PP5 & 274.4 & 342.6 & 28.50 & 23.44 & 17.53
\end{tabular}

${ }^{a} T_{\text {onset }}$ is the temperature at $5 \%$ weight loss and $T_{\max }$ is the temperature at maximum rate of weight loss. similar decomposition behavior in one-stage decomposition from 400 to $500{ }^{\circ} \mathrm{C}$, as shown in Fig. 3(a) and (b). This may be ascribed to the degradation of PP skeleton. The incorporation of PMGO leads to the decomposition of PP composites at lower temperature and the remarkable increase of the char residues from $7.12 \mathrm{wt} \%$ for PP2 (25 wt\% IFR/0 wt\% PMGO) to $16.10 \mathrm{wt} \%$ for PP5 (20 wt\% IFR/5 wt\% PMGO). In addition, it is also noted that the char yield of FR PP composite at above $500{ }^{\circ} \mathrm{C}$ presents a significantly increasing tendency with increase in PMGO content under nitrogen atmospheres. In DTG curves of various samples (Fig. 3(b)), with increasing PMGO content, the maximum thermal decomposition rate of FR PP sample obviously decreases, indirectly indicating that the flame retardancy of material will become much better. This meanwhile shows that the incorporated PMGO has the obviously synergistic effect. Comparatively, under air atmosphere, FR PP composites show a two-step degradation behavior, which is in the range of 250-400 ${ }^{\circ} \mathrm{C}$ and $400-700{ }^{\circ} \mathrm{C}$, respectively. It is noted that FR PP samples start to degrade at $266.8{ }^{\circ} \mathrm{C}$ and the decomposition basically ends at $700{ }^{\circ} \mathrm{C}$ with the char residue of about $12.65 \%$ (PP2). All compounded formulations show a higher decomposition temperature and higher char yield under air atmosphere than under nitrogen atmosphere before $550{ }^{\circ} \mathrm{C}$, which is possibly due to the oxygen shielding by the enhanced charred layers formed from the degradation of the incorporated IFR additives containing PMGO under air atmosphere. The related DTG curves also show the similar behavior to those under nitrogen atmosphere. This means that the addition of PMGO is also effective in enhancing the quality of char residues of IFRs under air atmosphere at high temperature.

Table 4 The calculated and experimental char yield of different samples obtained from TG measurement at $700{ }^{\circ} \mathrm{C}$ under nitrogen atmosphere

\begin{tabular}{llllllllr}
\hline Char yield & IFR & PMGO & PP0 & PP1 & PP2 & PP3 & PP4 & PP5 \\
\hline Calculated (\%) & - & - & - & 3.52 & 4.33 & 4.75 & 5.59 & 7.27 \\
Experimental (\%) & 16.49 & 58.5 & 0.28 & 5.80 & 7.12 & 7.86 & 12.83 & 16.10
\end{tabular}

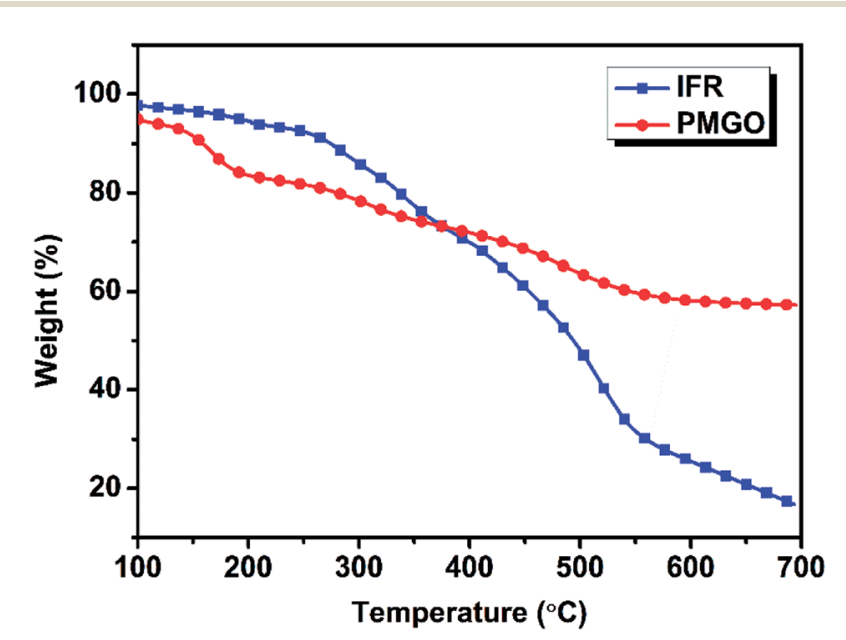

Fig. 4 The TGA curves of PMGO and IFR under nitrogen atmosphere. 

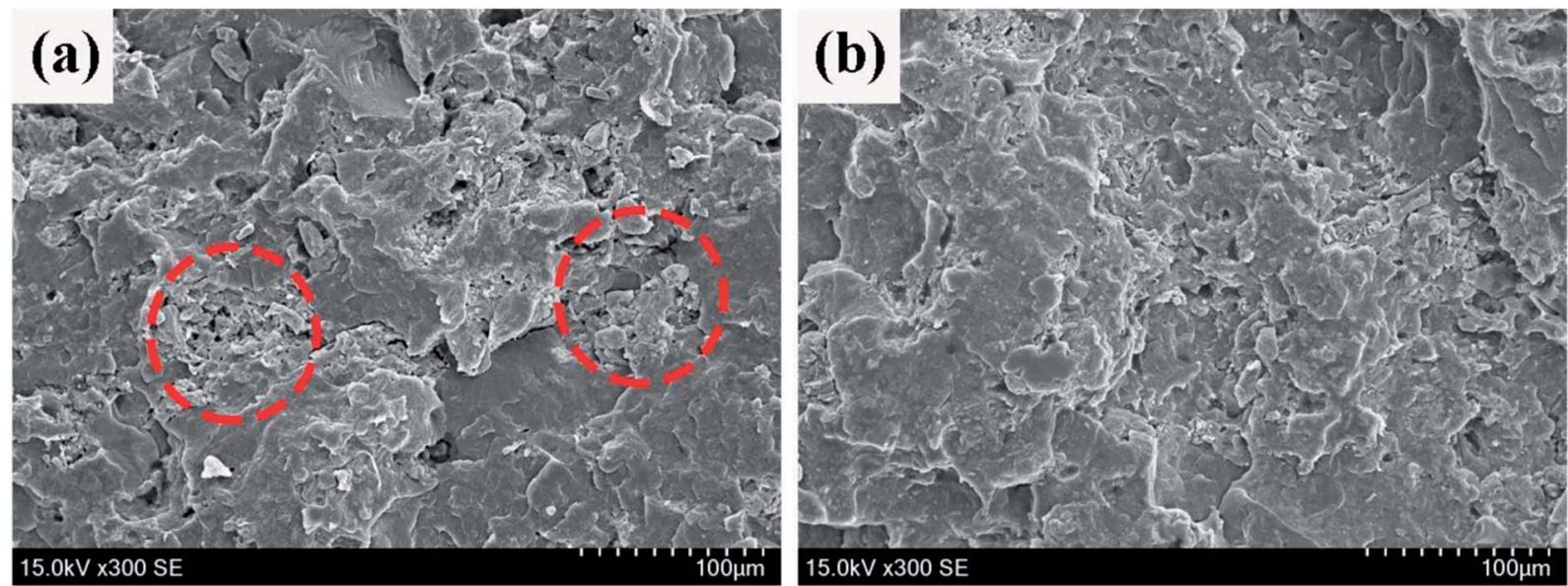

Fig. 5 The SEM photos of the fractured surface of FR PP composites: (a) PP2 and (b) PP5

In order to further investigate the synergistic effect between PMGO and IFR in FR PP composite, the calculated and experimental char yields of different samples at $700{ }^{\circ} \mathrm{C}$ (Table 4) based on the TG analysis results of PMGO and IFR under nitrogen (Fig. 4) were provided for comparison. It can be seen that the experimental char yield of all FR PP samples with $25 \mathrm{wt} \%$ FR loading (PP3 7.86\% to PP5 16.10\%) is much higher than the calculated one (PP3 4.75\% to PP5 7.27\%) correspondingly. Particularly, with increase in PMGO content in system, the difference between experimental value and calculated one also

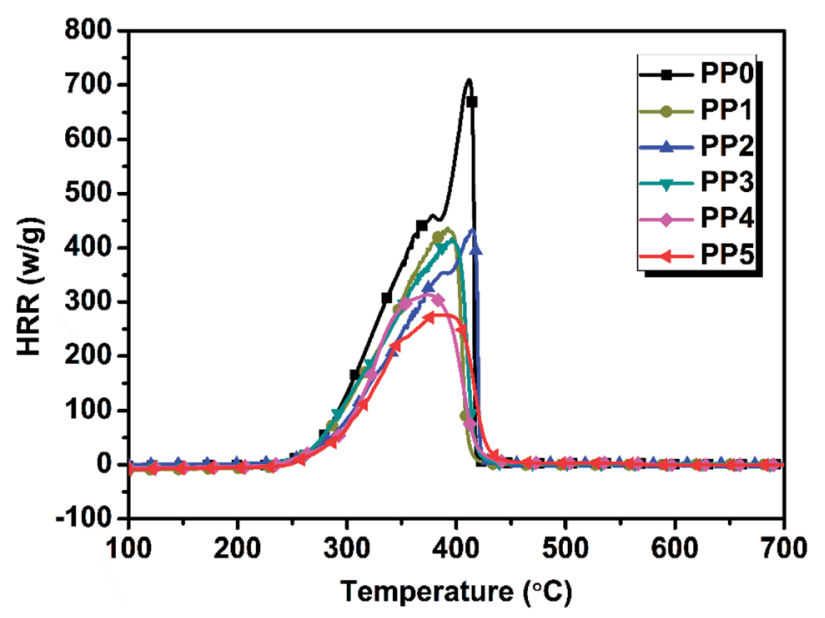

Fig. 6 MCC analysis curves of various FR PP composites.

Table 5 The MCC parameters of various FR PP composites $^{a}$

\begin{tabular}{lll}
\hline Sample & PHRR $\left(\mathrm{W} \mathrm{g}^{-1}\right)$ & THR $\left(\mathrm{kJ} \mathrm{g}^{-1}\right)$ \\
\hline PP0 & 715.6 & 43.3 \\
PP1 & 425.8 & 32.0 \\
PP2 & 422.6 & 31.7 \\
PP3 & 406.1 & 34.0 \\
PP4 & 313.5 & 27.8 \\
PP5 & 275.8 & 25.9
\end{tabular}

${ }^{a}$ PHRR is peak heat release rate and THR is total heat release. increases. This indicates that there are strong interactions between IFR and PMGO incorporated in PP matrix. The incorporated PMGO synergist promotes the carbonization of FR PP system. This also really shows that there is presence of synergism between PMGO and IFR.

SEM is a useful tool to well investigate the dispersion of fillers in polymer matrix. The SEM photos of the fractured surface of PP2 (without PMGO synergist) and PP5 (with 5 wt\% PMGO synergist) are shown in Fig. 5. It can be seen that there are heavily agglomerated flame retardant particles in PP2 sample. Comparatively, the dispersion and compatibility of flame retardant particles is obviously improved in PP5 sample with PMGO synergist. It seems that the presence of PMGO is possibly beneficial to the improvement of flame retardant dispersion and compatibility, which is very interesting and needs the further investigation later. This is also one of the reasons for the enhanced flame retardancy in PMGO synergist incorporated system.

\subsection{Flame retarding properties of FR PP composites}

3.3.1 MCC results. The flammability of various FR PP composites was evaluated by using micro-scale combustion calorimeter. The test results are shown in Fig. 6 and the corresponding MCC flammability parameters including peak heat release rate (PHRR) and total heat release (THR) for FR PP systems with different IFR/PMGO ratio are shown in Table 5 . Evidently, among all samples, pure PP shows the highest PHRR value and HRR value, achieving $715.6 \mathrm{~W} \mathrm{~g}^{-1}$ and $43.3 \mathrm{~kJ} \mathrm{~g}^{-1}$, respectively. When adding $20 \mathrm{wt} \%$ and $25 \mathrm{wt} \%$ loading of IFR

Table 6 The flammability tests of various samples

\begin{tabular}{llll}
\hline Sample & LOI $(\%)$ & UL94 rating & Flaming drips \\
\hline PP0 & 18.0 & NR & Yes \\
PP1 & 25.5 & NR & Yes \\
PP2 & 26.1 & NR & Yes \\
PP3 & 26.3 & NR & Yes \\
PP4 & 27.4 & V-1 & No \\
PP5 & 28.6 & V-0 & No
\end{tabular}




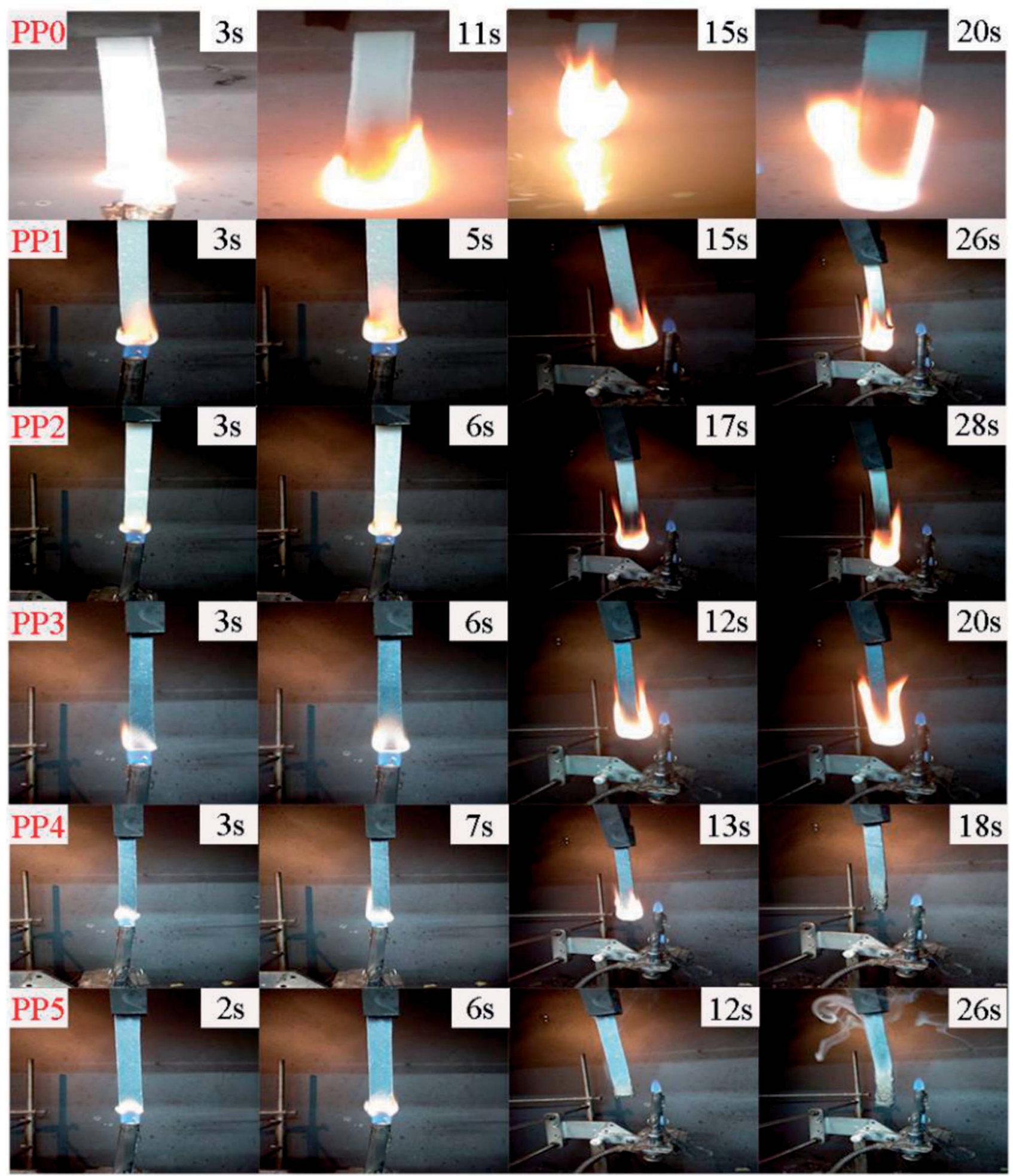

Fig. 7 The digital photos of PP0, PP1, PP2, PP3, PP4 and PP5 FR composites at different time during vertical burning test.

alone, the corresponding PHRR would decrease to $425.8 \mathrm{~W} \mathrm{~g}^{-1}$ (PP1) and $422.6 \mathrm{~W} \mathrm{~g}^{-1}$ (PP2), respectively. The related HRR curves exhibit the weakened peaks. Compared with PP2 and PP1, PP3 presents an abnormal increase in THR value $(34.0 \mathrm{~kJ}$ $\mathrm{g}^{-1}$ ). This could be possibly due to the graphene dispersion problem and also less content of graphene sheets that fail to play an effective physical barrier and block effect. As PMGO and IFR are combined together, it was worthy noted that the PHRR of PP5 with $5 \mathrm{wt} \%$ PMGO synergist becomes $275.8 \mathrm{~W} \mathrm{~g}^{-1}$, decreasing by $61.5 \%$ and $34.7 \%$ relative to neat PP and PP2 without PMGO, respectively. These results suggest that the highly graphitized chars formed from PMGO can effectively 

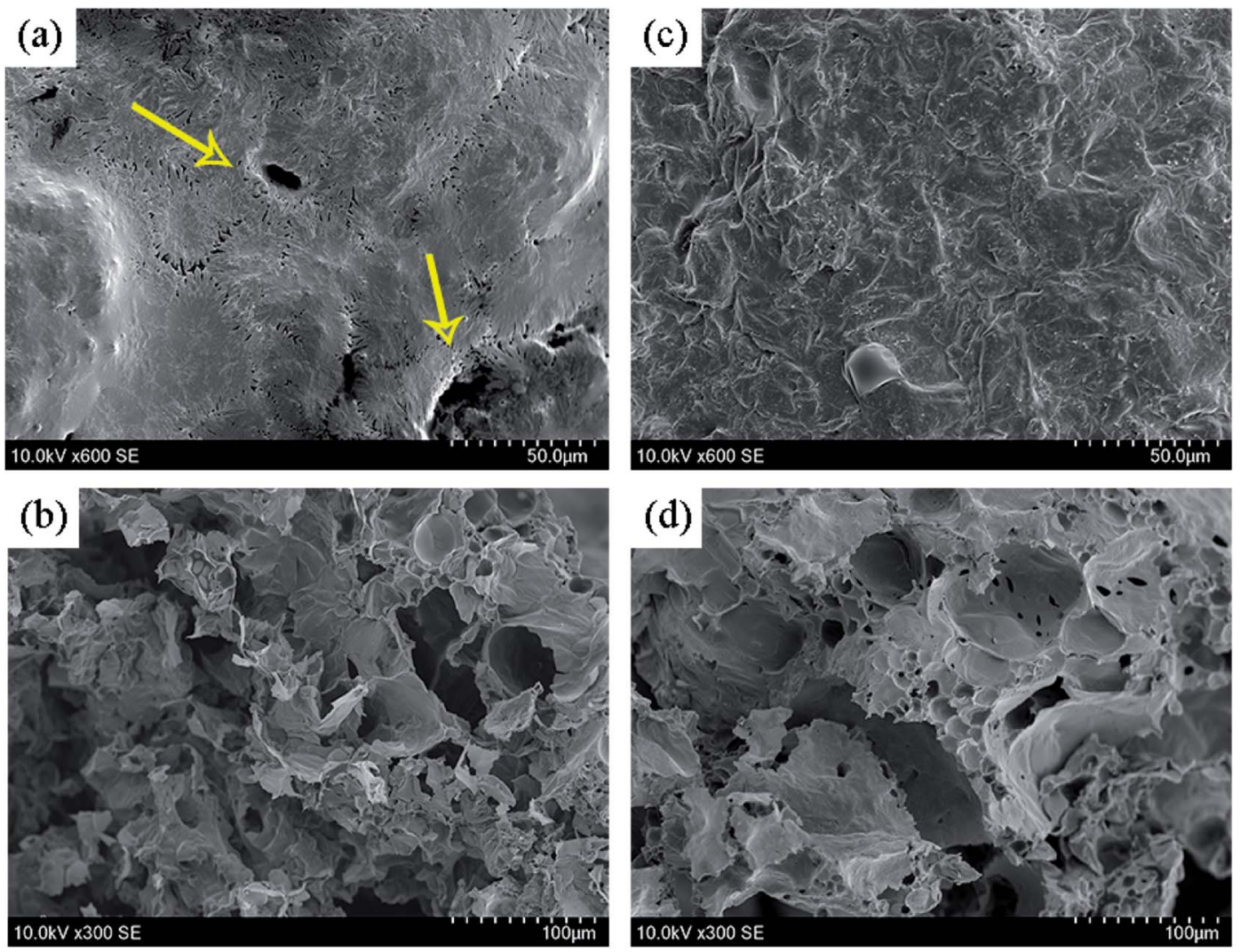

Fig. 8 SEM images of the expanded char layers of PP2 ((a)-outer side and (b)-inner side) and PP5 ((c)-outer side and (d)-inner side) samples after UL94 vertical burning test.

prevent the burning and protect the underlying PP polymer matrix from further burning. This means that the incorporated PMGO surely presents an effectively synergistic effect and contributes to a remarkable reduction in PHRR value of PP5.

3.3.2 UL94 and LOI flammability tests. Table 6 summarizes the UL94 and LOI results of all FR formulations. The corresponding digital photos of different samples during UL94 vertical burning test are shown in Fig. 7. Obviously, PP could not pass the UL94 test and only achieves a low LOI value of $18 \%$. Besides, it is clearly seen that PP0 burns vigorously with heavy flaming drips, which is very dangerous and easy to cause "secondary damage". When $20 \mathrm{wt} \%$ and $25 \mathrm{wt} \%$ loading of only IFR is incorporated, the corresponding LOI value of FR PP composite increases to $25.5 \%$ (PP1) and $26.1 \%$ (PP2), respectively and the burning rate of material is also slightly reduced. With introduction of PMGO synergist into PP/IFR system, the substrate of FR PP composite is found to be quickly expanded and carbonized, effectively suppressing the flame propagation within $12 \mathrm{~s}$ for PP5 sample containing $5 \mathrm{wt} \%$ PMGO synergist. Correspondingly, the LOI value of PP5 is further increased to $28.6 \%$. Even after the second flame application, PP5 could also extinguish promptly after $26 \mathrm{~s}$.

\subsection{Morphology analysis of char residues}

High-quality char layer formed in condense phase plays a very critical role in improving the flame retardancy of polymer material. Fig. 8 presents the digital photos of internal and external carbon layers of PP2 and PP5 after UL94 vertical burning tests. No residues are left after burning test, which directly illustrates the strong flammability of pure PP. Fig. 8(a) and (b) show the morphologies of char layers of PP2 sample. It can be seen that there are some obvious cracks and crevasses observed on the exterior surface of char layers. In the inner surface of char layers, there are also many porous thin-walled structures found. Part of the cracks and holes are connected each other between the inner side and the outer side. Obviously, such a structure of carbon layers would surely lead to poor flame retardancy. Comparatively, as shown in the Fig. 8(c) and (d), the addition of $5 \mathrm{wt} \%$ PMGO leads to the distinct enhancement in the compactness of char layers of PP5 FR composite at both outer side and inner side. The carbonization of PMGO during material combustion makes it not only provide an effective silicon-based protective layers on the graphene surface from oaPOSS, but also generate the thermal insulation layers from the graphene sheet itself and phosphorus-containing groups in DOPO, which can effectively improve the strength of the graphene sheets dispersed in the expanded carbon layers formed from IFR. As a result, the rigidity and compactness of the final expanded carbon layers can be substantially enhanced. This implies that the synergistic effect existing between PMGO and IFR would result in formation of high-quality and homogenous char layers. The combination of the LOI and UL94 vertical burning test results can well illustrate the synergistic effect of PMGO on IFR in improving the flame-retarding properties of FR PP composites. It demonstrates that the optimum FR loading is the combination of $5 \mathrm{wt} \%$ PMGO and $20 \mathrm{wt} \%$ IFR used in PP 

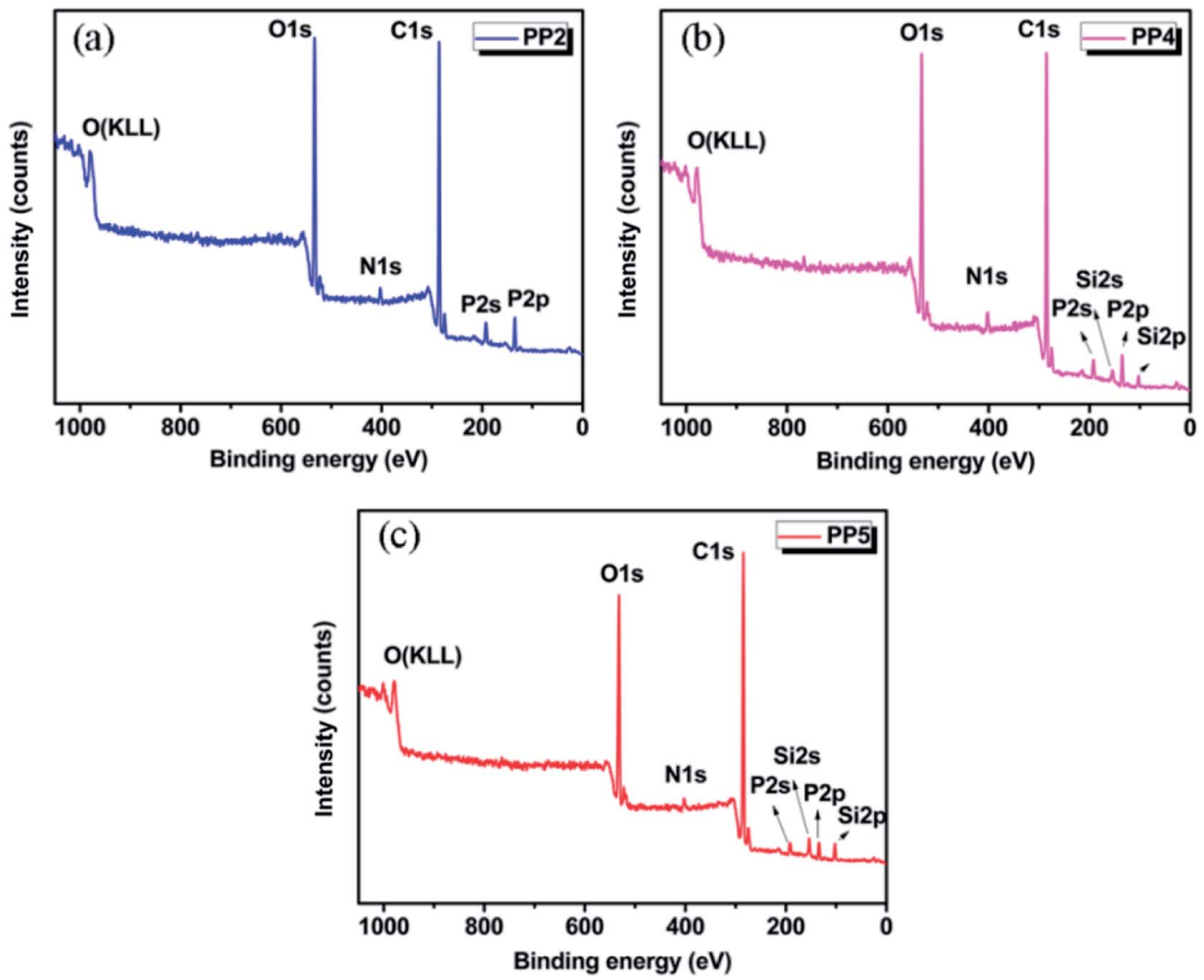

Fig. 9 The XPS survey spectra of char residue of PP2 (a), PP4 (b) and PP5 (c) after UL94 vertical burning test.

Table 7 The XPS results of various FR PP composites after UL94 vertical burning test

\begin{tabular}{llllll}
\hline Sample & C (at\%) & O (at\%) & N (at\%) & P (at\%) & Si (at\%) \\
\hline PP2 & 64.73 & 28.44 & 2.41 & 4.42 & - \\
PP4 & 67.30 & 25.41 & 2.79 & 3.42 & 1.09 \\
PP5 & 68.86 & 23.89 & 2.05 & 2.57 & 2.62
\end{tabular}

composite in view of its significantly improved flame retardancy and char residue.

\subsection{XPS analysis of char residues}

As we know, X-ray photoelectron spectroscopy (XPS) can provide further information to investigate the flame retarding mechanism and the elemental content of char residue in detail, which could be used as the supplement to the results from SEM observation. The XPS survey spectra of C, O, N, P and Si of char residue of various $\mathrm{PP} / \mathrm{IFR} / \mathrm{PMGO}$ samples after the combustion test are shown in Fig. 9. The corresponding surface element content is displayed in Table 7. With increasing PMGO loading, the content of $\mathrm{C}$ increases slightly because the formation of stable $\mathrm{C}$ structures $(\mathrm{C}-\mathrm{C} / \mathrm{C}=\mathrm{C})$ occurs in PMGO. The decrease in $\mathrm{O}$ content may be due to the water release, the release of other gases and the formation of $\mathrm{C}-\mathrm{O} / \mathrm{P}-\mathrm{O} / \mathrm{Si}-\mathrm{O}$ structures during burning. The content of $\mathrm{N}$ has the parallel change tendency, which may be ascribed to the lower loading of IFR and release of inert gases like $\mathrm{NH}_{3} / \mathrm{N}_{2}$. With increase of PMGO content from PP2 to PP5, it is found that the surface silicon content would also increase. This is because for the introduced PMGO, the rigid $\mathrm{Si}-$ O-Si structure is grafted on the surface of GO, which could further enhance the intumescent carbon layers and provide PP matrix with an extra enhanced protective covering. This is showing the excellent synergistic effect of PMGO. Therefore, PP5 exhibits a better flame retardancy than all other materials.

Based on the above characterizations and analyzes on the charred layers, a possible flame retarding mechanism for IFR/ PMGO synergistic system in FR PP composites is proposed in Scheme 2. During the combustion of FR PP composites, the grafted oapPOSS is thermally oxidized into a rigid silicon reinforced protective layer covered on GO layer surface and the grafted DOPO is also decomposed into a phosphorus-containing barrier layer covered on GO layer surface. Above two factors are both advantageous to enhancement in the stability and compactness of the graphene sheets, thus effectively improving the quality of the carbon layers that formed from the IFR. Such the hybrid intumescent charred layers with high strength and increased thermaloxidative stability can act as an effectively physical barrier to reduce the mass and heat transfer between the gas phase and condensed phase and can also prevent the escape of organic volatiles, thus delaying the heat release and protecting the underlying polymer 


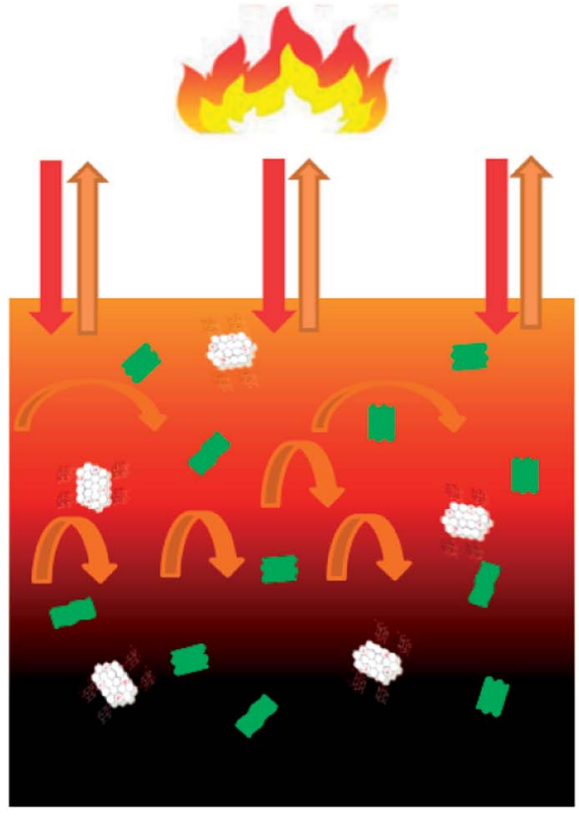

FR PP composites

IFR Heat and radiation

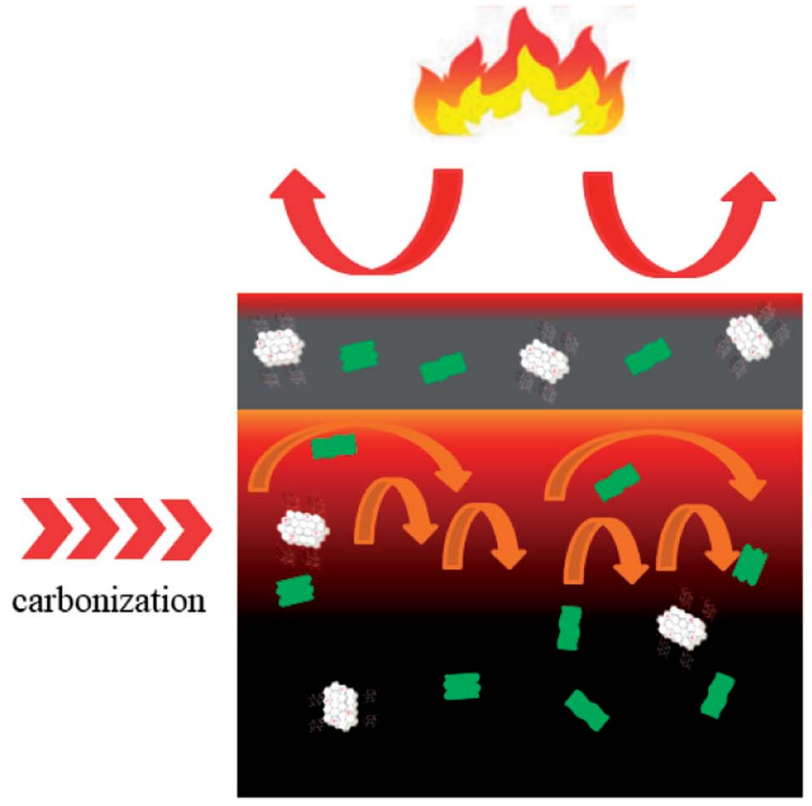

FR PP composites

PMGO

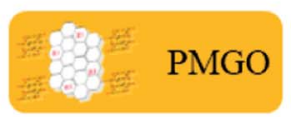

Scheme 2 The schematic diagram of the proposed flame-retarding mechanism for IFR/PMGO incorporated FR PP composites.

from fire. As a result, a distinct improvement of the fire safety of FR PP composites can be achieved.

\subsection{Wettability property of FR PP composites}

Water contact angle (WCA) measurement was employed to investigate the hydrophilic and hydrophobic property of FR PP composite. As depicted in Fig. 10, pure PP displays a good hydrophobicity with a WCA of $93.6^{\circ}$. However, a significant reduction in hydrophobicity is observed in only IFR incorporated PP composites (PP1 and PP2), where the minimum WCA of $63.9^{\circ}$ occurs at PP2 sample with $25 \mathrm{wt} \%$ loading of IFR. This means that the compatibility of FR PP system becomes poorer to some extent. The main reason for this could be explained by a large number of polar functional groups in IFR. This adverse factor may limit the versatility of FR PP material for applications such as in humid environments. Interestingly, with increasing PMGO content (PP2 $\rightarrow$ PP5), the WCA of the corresponding FR PP composite exhibits an obvious
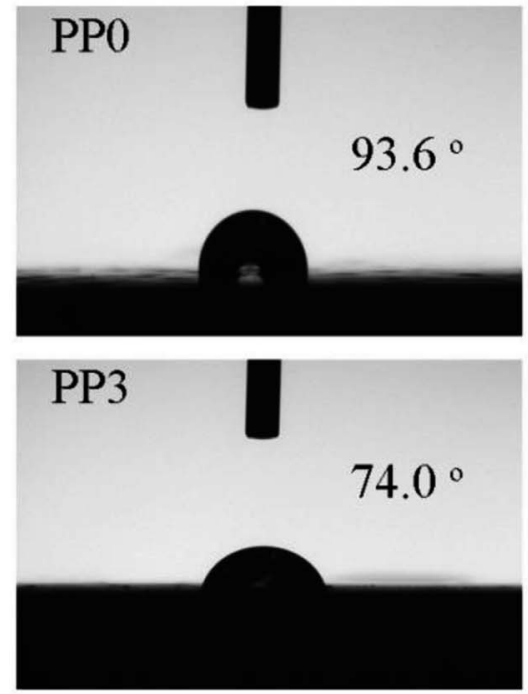
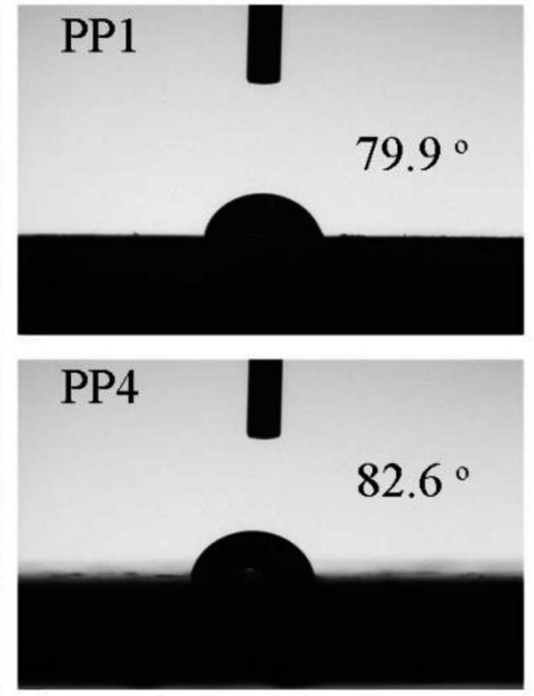
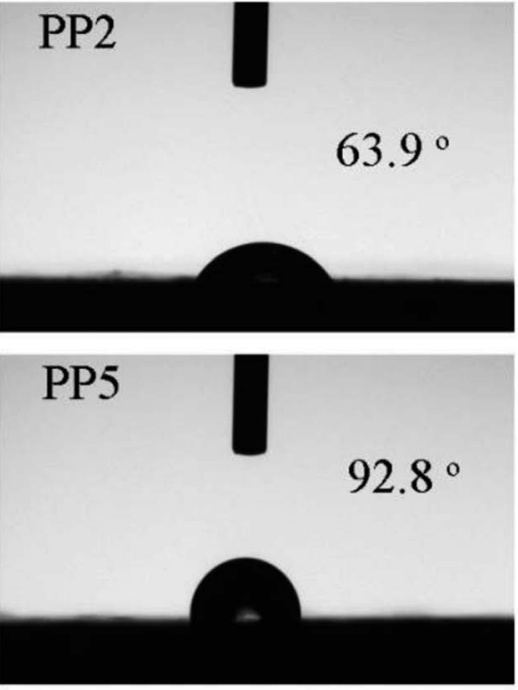

Fig. 10 The digital photos of water contact angle for different FR PP composites. 
increase tendency. Particularly, it is noted that PP5 sample achieves the best surface hydrophobicity with a WCA of $92.8^{\circ}$, which could be possibly caused by the herein hydrophobic property of graphene network structure on one hand. On the other hand, adopting oapPOSS for surface modification of GO can lead to the enhanced hydrophobicity because the POSS derivatives was reported to be the effective hydrophobic agents. $^{39,40}$ This is also possibly beneficial to the improvement in the compatibility and hence the mechanical performance of FR PP composite from another point of view.

\section{Conclusions}

In this work, a novel graphene oxide based flame retardant (PMGO) integrating phosphorous, nitrogen and silicon element contained structures together were synthesized through in situ condensation reaction. The FT-IR and XPS measurements confirmed the successful synthesis of PMGO. The prepared PMGO was subsequently formulated with the traditional intumescent flame retardants as a synergist to flame-retard PP. The synergistic flame retardancy of the obtained FR PP composites were investigated. The TGA results demonstrate that FR PP composites with $20 \mathrm{wt} \%$ IFR and $5 \mathrm{wt} \%$ PMGO is more efficient than those with only IFR (25 wt\%) alone in enhancing the thermal-oxidative stability of PP system in high temperature range (above $500{ }^{\circ} \mathrm{C}$ ) and increasing the amount of residual chars. Besides, the flammability tests also show the synergistic effect of PMGO on the IFR incorporated PP system. The PHRR and THR of the final FR PP composites are reduced $61.5 \%$, $40.2 \%$, respectively, compared with neat PP. Also, the prepared FR PP composites achieve the LOI value of $28.6 \%$ and UL94 rating of V-0 level, which means a substantial improvement in the flame retardancy of PP. The char residues of FR PP composites after UL94 vertical flammability test were systematically characterized using SEM and XPS measurements and the possible mechanism of synergistically flame-retarding PP with IFR/PMGO formulation was proposed accordingly. Finally, the water contact angle measurement shows that the obtained FR PP composites maintain a good hydrophobic property, which could broaden its range of applications in humid environments.

\section{Conflicts of interest}

There are no conflicts of interest to declare.

\section{Acknowledgements}

This work is kindly supported by the National Key Research and Development Program of China (2016YFB0302101), the National Natural Science Foundation of China (51721091) and the Program of Innovative Research Team for Young Scientists of Sichuan Province (2016TD0010).

\section{References}

1 X. Li, T. Yan, X. Hu, K. Wang and Q. Fu, Polym. Eng. Sci., 2013, 53, 410-416.
2 Y. Chen, Z. Guo and Z. Fang, Polym. Eng. Sci., 2012, 52, 390398.

3 M. Doğan and E. Bayraml, Polym. Adv. Technol., 2011, 22, 1628-1632.

4 Y. Han, T. Wang, X. Gao, T. Li and Q. Zhang, Composites, Part A, 2016, 84, 336-343.

5 Z. X. Zhang, J. Zhang, B. X. Lu, Z. X. Xin, C. K. Kang and K. K. Jin, Composites, Part B, 2012, 43, 150-158.

6 M. K. A. Sofiah, O. H. Lin, H. M. Akil and Z. A. M. Ishak, Mater. Sci. Forum, 2014, 803, 265-268.

7 S. Pappalardo, P. Russo, D. Acierno, S. Rabe and B. Schartel, Eur. Polym. J., 2016, 76, 196-207.

8 T. Jiang, C. Liu, L. Liu, J. Hong, M. Dong and X. Deng, RSC Adv., 2016, 6, 91720-91727.

9 Y. Liu and Q. Wang, Polym. Degrad. Stab., 2006, 91, 25132519.

10 F. Qi, M. Tang, N. Wang, N. Liu, X. Chen, Z. Zhang, K. Zhang and X. Lu, RSC Adv., 2017, 7, 31696-31706.

11 L. Lu, N. Guo, X. Qian, S. Yang, X. Wang, J. Jin and G. Shao, J. Appl. Polym. Sci., 2017, 45962.

12 B. Yuan, A. Fan, M. Yang, X. Chen, Y. Hu, C. Bao, S. Jiang, Y. Niu, Y. Zhang, S. He and H. Dai, Polym. Degrad. Stab., 2017, 143, 42-56.

13 X. Qian, L. Song, Y. Bihe, B. Yu, Y. Shi, Y. Hu and R. K. K. Yuen, Mater. Chem. Phys., 2014, 143, 1243-1252.

14 Z. Zheng, X. Cui and H. Wang, Polym. Compos., 2015, 36, 1606-1619.

15 Y. Zhang, X. Chen and Z. Fang, J. Appl. Polym. Sci., 2013, 128, 2424-2432.

16 X. Almeras, M. L. Bras, P. Hornsby, S. Bourbigot, G. Marosi, S. Keszei and F. Poutch, Polym. Degrad. Stab., 2003, 82, 325331.

17 A. Riva, G. Camino, L. Fomperie and P. Amigouët, Polym. Degrad. Stab., 2003, 82, 341-346.

18 Z. Zheng, Y. Liu, L. Zhang and H. Wang, J. Mater. Sci., 2016, 51, 5857-5871.

19 H. Kim, A. A. Abdala and C. W. Macosko, Macromolecules, 2010, 43, 6515-6530.

20 A. K. G. K. S. Novoselov, Nat. Mater., 2007, 6, 183-191.

21 A. K. G. K. S. Novoselov, S. V. Morozov, D. Jiang, Y. Zhang, S. V. Dubonos, I. V. Grigorieva and A. A. Firsov, Science, 2004, 306, 666-669.

22 L. M. Veca, M. J. Meziani, W. Wang, X. Wang, F. Lu, P. Zhang, Y. Lin, R. Fee, J. W. Connell and Y.-P. Sun, Adv. Mater., 2009, 21, 2088-2092.

23 S. G. A. A. Balandin, W. Bao, I. Calizo, D. Teweldebrhan, F. Miao and C. N. Lau, Nano Lett., 2008, 8, 902-907.

24 X. W. Changgu Lee, J. W. Kysar and J. Hone, Science, 2008, 321, 385-388.

25 G. Huang, J. Gao, X. Wang, H. Liang and C. Ge, Mater. Lett., 2012, 66, 187-189.

26 B. Yu, Y. Shi, B. Yuan, S. Qiu, W. Xing, W. Hu, L. Song, S. Lo and Y. Hu, J. Mater. Chem. A, 2015, 3, 8034-8044.

27 S. Stankovich, R. D. Piner, S. T. Nguyen and R. S. Ruoff, Carbon, 2006, 44, 3342-3347.

28 H. Bai, C. Li and G. Shi, Adv. Mater., 2011, 23, 1089-1115. 
29 R. Sengupta, M. Bhattacharya, S. Bandyopadhyay and A. K. Bhowmick, Prog. Polym. Sci., 2011, 36, 638-670.

30 G. Huang, S. Chen, S. Tang and J. Gao, Mater. Chem. Phys., 2012, 135, 938-947.

31 W. Gao, L. B. Alemany, L. Ci and P. M. Ajayan, Nat. Chem., 2009, 1, 403-408.

32 H. J. Salavagione, M. n. A. Gómez and G. Martínez, Macromolecules, 2009, 42, 6331-6334.

33 W. Chen, Y. Liu, P. Liu, C. Xu, L. Yuan and W. Qi, Sci. Rep., 2017, 7, 8759.

34 F. Sun, T. Yu, C. Hu and Y. Li, Compos. Sci. Technol., 2016, 136, 76-84.
35 S.-H. Liao, P.-L. Liu, M.-C. Hsiao, C.-C. Teng, C.-A. Wang, M.-D. Ger and C.-L. Chiang, Ind. Eng. Chem. Res., 2012, 51, 4573-4581.

36 X. Wang, Y. Hu, L. Song, H. Yang, B. Yu, B. Kandola and D. Deli, Thermochim. Acta, 2012, 543, 156-164.

37 P. Zong, J. Fu, L. Chen, J. Yin, X. Dong, S. Yuan, L. Shi and W. Deng, RSC Adv., 2016, 6, 10498-10506.

38 Z. Zhang, G. Liang and T. Lu, J. Appl. Polym. Sci., 2007, 103, 2608-2614.

39 A. Wamke, J. Makowiecki, K. Dopierała, J. Karasiewicz and K. Prochaska, Appl. Surf. Sci., 2018, 443, 280-290.

40 E. R. Smith, B. J. Howlin and I. Hamerton, J. Mater. Chem. A, 2013, 1, 12971-12980. 